\title{
Disentangling pediatric bipolar disorder and attention deficit-hyperactivity disorder: A neuropsychological approach
}

\author{
F. Zaravinos-Tsakos, G. Kolaitis \\ Department of Child Psychiatry, School of Medicine, National and Kapodistrian University of Athens, \\ "Aghia Sophia" Children's Hospital, Athens, Greece
}

Psychiatriki 2020, 31:332-340

\begin{abstract}
he clinical and diagnostic debate circulating pediatric bipolar disorder (PBD) has been highlighted as one of the most controversial themes in child psychiatry. With atypical symptomatic expression, constituting its predominant diagnostic discrepancy, PBD is manifested through prolonged manic episodes and affective storms, lacking the symptomatic cycling and episodic nature presented in adult $\mathrm{BD}$. Apart from its unique clinical presentation, the substantial symptomatic overlap with attentiondeficit hyperactivity disorder (ADHD) indicate an important diagnostic challenge in PBD. Specifically, both disorders share core characteristics such as irritability, hyperactivity, excessive talking and distractibility. Against this background of findings on the overlapping symptomatology between PBD and ADHD, current research guidelines highlight the need of exploring non-symptomatic markers as potential clinical phenotypes. Especially in disorders with distinctive biologic underpinnings, both clinicians and researchers have shown increased interest in establishing neuropsychological profiles. Recent neuropsychological studies indicated the distinct nature of neurocognitive deficits in PBD, describing impairments in various cognitive skills during acute episodes phases, while this severe deterioration of cognitive deficits appears to persist even during euthymic states. Regarding neuropsychological assessment in AHD, recent findings suggested dysfunctions in the domains of working memory, verbal memory and response inhibition. Furthermore, neuroimaging studies are fast becoming a key instrument to establish distinct neuropsychological profiles for PBD and ADHD. A large number of neuroimaging studies have indicated abnormalities in limbic, cortical and subcortical brain systems, while meta-analytic findings of voxel based morphometric studies highlight abnormalities in dorsolateral and lateral orbitofrontal-temporal areas in PBD. In recent neuroimaging findings with focus on neurocognitive performance during an emotional Stroop task, patients diagnosed with ADHD indicated activation on higher cortical centres associated with processing speed and significantly decreased role of sustained attention. Furthermore, these findings suggest emotional regulation and inhibitory control are moderately intercorrelated, adding more complexity to the theme of neurocognitive deficits in ADHD. These observations on the neurobiological mechanisms of cognitive impairments in PBD
\end{abstract}


appear to provide robust evidence on a potential specific neuropsychological profile of PBD, the relashionship between mood states and neuropsychological functioning, and the link between emotion generation and regulation in children with PBD.

Key words: Bipolar disorder, attention-deficit hyperactivity disorder, neuropsychological assessment, diagnosis.

\section{Introduction}

Over the past decades, rapid advances have been witnessed in the field of pediatric bipolar disorder (PBD). The predominant view that PBD is almost non-existent has been challenged by a considerable amount of case reports and case series. ${ }^{1-6}$ However, difficulties arose regarding the diagnostic profile of children with PBD. As noted by Weller, ${ }^{7}$ PBD may be common among referred children with severe psychopathology but it is also associated with several diagnostic discrepancies.

These difficulties in the diagnosis of PBD were the primary focus of related research in later years. PBD is characterized as atypical in comparison with bipolar disorder (BD) in adults ${ }^{8}$ since its clinical course is manifested through continuous affective storms and prolonged manic and irritable manic outbursts, in contrast with the regular fluctuation and/or rapid cycling between mood states in adults diagnosed with BD. ${ }^{9-10}$ Although a growing body of current research provides substantial evidence in overcoming the phenomenological and clinical disagreements of $\mathrm{PBD}^{11-12}$ there is an increasing concern about a specific diagnostic issue that appears to regulate the establishment of PBD. In particular, the symptom overlap between PBD and attention-deficit hyperactivity disorder (ADHD) has become a new issue for debate between clinicians and researchers. ${ }^{13}$

\section{Symptomatic overlap in PBD and ADHD}

The debate concerning PBD and ADHD has attracted growing attention for various reasons. In terms of clinical course, PBD and ADHD indicate an amassing overlap of symptoms. Although pivotal clinical characteristics of PBD such as chronic and continuous severe irritability and prolonged aggressive temper outbursts appear to form a distinct diagnostic type, various systematic studies of children and adolescents show that rates of ADHD range from $60 \%$ to $90 \%$ in children diagnosed with PBD..$^{14-15}$ Moreover, PBD and
ADHD share core characteristics such as excessive talking, hyperactivity, inappropriate actions and verbal responses in social situations, lack of inhibition, and distractibility; it is of great importance that these symptoms are common in both manic and hypomanic phases of PBD. ${ }^{16}$ Furthermore, chronic irritability, one of the hallmark features of childhood mania, is transparently manifested through emotional liability and low frustration tolerance in ADHD., ${ }^{9,16}$ A striking finding is that even when eliminating the overlapping items of PBD, the diagnosis of mania is still plausible while in case of eliminating overlapping symptoms of ADHD, such as inattention, hyperactivity, impulsiveness, and irritability are eliminated, the disorder itself disappears. ${ }^{17}$ This paradox is also indicated by the fluctuation of the prevalence rates for PBD. According to recent meta-analytic findings based on outpatient and inpatient samples, prevalence rates of PBD range from $0 \%$ in the UK, $0.006 \%$ in Finland, $1.2 \%$ in Denmark, $1.9 \%$ in the Netherlands, $4 \%$ in Spain, $4.2 \%$ in India and 6\% in the US18. Consequently, the PBD spectrum which includes bipolar I, bipolar II and cyclothymic disorder affects between $0 \%$ and $6 \%$ of children in general population, averaging $1.8 \%$ worldwide ${ }^{18-19}$ while ADHD is far more common, averaging in $5.3 \% .^{20}$ These contradictory findings are reflected on the true comorbidity estimates for both disorders, excluding overlapping symptoms with $2 \%$ of ADHD cases would be expected to have PBD, $8 \%$ of PBD cases would have ADHD symptoms while only 3 in 2.000 children would have both disorders. ${ }^{21}$ Another finding that adds to the general confusion is that the rates of comorbidity tend to be elevated exclusively in clinical populations. ${ }^{22}$

\section{Diagnostic discrepancies in PDB and ADHD}

In light of recent findings regarding the ADHD symptoms in PBD, diagnosis of the latter is becoming increasingly difficult in children. Although a considerable amount of literature has already grown up on psychopathological comorbidity in children, ${ }^{23-24}$ 
the debate on PBD has gained fresh prominence with the comorbid symptoms of PBD and ADHD25. In terms of diagnosis, one major issue that dominates the debate is the overlapping nature of the diagnostic criteria for PBD and ADHD, which heavily relies on irritability and distractibility. ${ }^{16,26}$ Despite being one of the most prominent symptoms of mania or hypomania at all ages, distractibility is of little utility in differential diagnosis in children because of its ubiquity across a considerable amount of childhood onset disorders such as major depressive disorder, ADHD, oppositional defiant/conduct disorders. ${ }^{15}$

In an attempt to resolve the problem of overlapping symptoms, various approaches have been adapted. In particular, it is speculated that ADHD might constitute a prodromal for PBD. ${ }^{27}$ However, while epidemiological studies indicate that an earlier onset for ADHD may be a precursor for $\mathrm{PBD}$, longitudinal studies of cohorts with ADHD have found that only a limited amount of ADHD cases were lately diagnosed with PBD28. Moreover, a considerable number of adults with $\mathrm{BD}$ never received a prior ADHD diagnosis. ${ }^{22}$ Furthermore, there is a notion that surveillance or referral biases have substantial impact on clinical findings of PBD. In particular, in a study conducted by Wozniak, ${ }^{9}$ all of cases ascertained from an ADHD clinical department also met criteria for PBD. In contrast, recent findings on cohort studies suggested significantly lower comorbidity rates between PBD and ADHD, indicating that the high estimate was due to recruitment patterns. For example, overrepresentation of boys in clinical samples is associated with more severe disruptive behavior and consequent predominant manic symptoms. ${ }^{29}$ Furthermore, various potential confounders may account for our distorted clinical image about the comorbidity between PBD and ADHD. Specifically, hypomanic children are commonly under-represented in clinical samples since hypomanic symptoms do not constitute dysfunctional attitudes to motivate help seeking. ${ }^{30}$ In addition, training and conceptualization differences between structured and semi-structured interviews used in pediatric and juvenile clinical samples, indicate an inadequate protection against referral and surveillance biases. What is surprising is that disagreements among clinicians remain even when predominant manic symptoms are evident in videotaped interviews or in identical case reports. ${ }^{31-32}$

\section{Neuropsychological impairments in PBD and ADHD}

Against this background of findings on overlapping features, empirical studies intending to disentangle PBD and ADHD through direct comparison are still sparse. The majority of current research has solely focused on the clinical differences in patients with comorbid PBD and ADHD or in children diagnosed with either PBD or ADHD. Remarkably, although empirical findings of neuropsychological impairments in PBD and ADHD indicate neurobiological differences between these disorders, neuropsychological studies contrasting PBD and ADHD are also lacking.

It is nowadays well established that various cognitive functions are impaired in children diagnosed with PBD. ${ }^{33}$ In particular, recent neuropsychological studies highlighted the pervasive and severe nature of cognitive impairments in acute episode phases. ${ }^{34,35}$ What is of great interest is that these deficits appear to persist even during euthymic states. ${ }^{36,37} \mathrm{~A}$ meta-analysis conducted by Walshaw ${ }^{38}$ including a large number of studies over the past two decades, indicated that children with PBD present deficits on various standardized neuropsychological measures, with significantly worse performance in executive function and verbal learning tasks. In accordance to previous findings, these deficits persist despite any bipolar symptom reduction. ${ }^{39}$ Moreover, according to these metaanalytic findings, euthymic states are characterized by moderate levels of neuropsychological impairment, with specific deficits in verbal learning and memory. Deficits in executive functions, such as response inhibition and set shifting, are also among the main neurocognitive impairments in euthymic bipolar populations. ${ }^{40}$ In contrast, only a subset of neurocognitive functions, such as visual memory, phonemic fluency, and motor skills, is associated with worse performance during acute episodes of the disorder. ${ }^{41}$

Regarding ADHD, existing neuropsychological research suggested impairments in processing speed, motor speed, verbal declarative memory. ${ }^{42,43}$ Moreover, executive functions, such as working memory and set-shifting, were found to suffer the most. ${ }^{44} \mathrm{~A}$ key point to neuropsychological profile of ADHD is the developmental nature of these cognitive dysfunctions. As highlighted in longitudinal 
studies, these deficits are persistent and stable..$^{45,46}$ Furthermore, the presence of stable brain abnormalities in children with ADHD supports the possibility for impaired neuropsychological performance in adult life. ${ }^{47}$ In terms of differences between ADHD subtypes, evidence suggests that both the inattentive and combined types share the same core deficits in executive functions, with the exception of response inhibition, in which a possible distinguishable profile was found in boys with the combined type. ${ }^{48}$

\section{Neuropsychological profiling as a diagnostic adjunctive?}

It has been established now that neurocognitive impairments in the bipolar spectrum are not moderated by temporary functional changes and are associated with stable functional alterations of specific neural networks. ${ }^{49}$ Consequently, various neurocognitive deficits, such as sustained attention, working and verbal memory, verbal and cognitive flexibility, have been reported in all age, manic and depressive, bipolar populations. Moreover, these findings suggest differences between the neurocognitive profiles of PBD and ADHD. In particular, children diagnosed with $\mathrm{BD}$ present a specific pattern of impairments in inference control, set-shifting and sustained attention, while children with ADHD are mostly impaired in verbal and spatial working memory and phonemic verbal fluency. ${ }^{38}$ Moreover, it is of great importance that these findings are in line with reported neurocognitive deficits in adult populations. In specific, studies suggest significantly poor performance in interference scores on the Stroop task and substantially more perseverative errors on the Winscoscin Card Sorting Test (WCST) of BD adults in comparison with healthy controls, ${ }^{50,51}$ while studies in the adult ADHD provide robust evidence on poor performance in working memory and phonemic fluency tasks. ${ }^{52,53}$ Based on this agreement between neurocognitive findings in juvenile and adult populations diagnosed with $\mathrm{BD}$ or ADHD, Walshaw ${ }^{38}$ states that "evidence for distinctive profiles of neurocognitive functions in these disorders exists". However, the concept of disentangling PBD from ADHD based on neurocognitive performance still needs to be established. In particular, the issue of the relationship between mood states and cognitive deficits in PBD provides a fruitful challenge for clinicians and researchers.

\section{Neuroimaging evidence in PBD and ADHD}

In light of these advances, neuroimaging studies are fast becoming a key instrument to resolve various limitations in neurocognitive research in BD. A substantial amount of neuroimaging studies has indicated abnormalities in limbic, cortical and subcortical brain systems ${ }^{54}$ while meta-analytic findings of voxel based morphometric studies highlight abnormalities in dorsolateral and lateral orbitofrontal-temporal areas (DLPFC) in clinical populations diagnosed with $\mathrm{BD}$. A striking finding is that these brain systemic abnormalities are associated with dysfunctional patterns of neural activation and suggest symptomspecific neurocognitive deficits in bipolar patients. In particular, depression-associated cognitive deficits in BD result from either limbic activation with hypometabolism of the DLPFC or hypo-activation of the ventral anterior cingulate (vACC), which accounts for hypo-arousal and anhedonia. ${ }^{41}$ In contrast, maniclike symptoms are expressed through increased activity of the left hemisphere prefrontal corticalsubcortical system and suggest heightened distractibility and behavioural dysregulation. ${ }^{55}$ Concerning neural activation differences between euthymic states and mood episodes, decreased performance on Stroop tasks highlights the role of ventral prefrontal cortex (vmPFC). When compared to a euthymic group, patients with mania present blunted activation on the right side of PFC while patients with bipolar depression exhibit substantial increase on the left side of the PFC. ${ }^{56}$ Furthermore, during workingmemory tasks, adolescent BD patients present significant activation of the anterior cingulate cortex (ACC), DLPFC and inferior frontal PFC in comparison with the reported hypo-activation in the same regions. ${ }^{57}$ In terms of performance during decisionmaking tasks, patients during manic episodes, have shown increased activation in dorsal ACC (dACC) along with concurrent hypo-arousal of the right PFC. Accordingly, dACC activation has been reportedly moderated by the severity of the manic episode. ${ }^{58}$

While these neuroimaging and neurocognitive results appear to establish a phenotypic profile for emotion regulation patterns in $\mathrm{BD}$, empirical studies in the same context for patients diagnosed with ADHD indicate significant inconsistencies. In particular, despite dysfunctions in the medial and ventrolat- 
eral prefrontal cortex are common in ADHD and BD, the localized circuits responsible for emotion regulation in ADHD appear to be not disorder-specific, since they are also met in disorders such as oppositional defiance disorder, conduct disorder and major depressive disorder. ${ }^{59}$ Moreover, questions have been raised about the role of deficient executive inhibitory control, which is regarded as the hallmark feature of impaired emotion generation and regulation in ADHD. Specifically, in recent neuroimaging findings which focused in neurocognitive performance during an emotional Stroop task, patients diagnosed with ADHD showed activation on higher cortical centers associated with processing speed and a significantly decreased role of sustained attention60. However, the most substantial drawback in the identification of specific neurocognitive patterns for emotion regulation in ADHD is the existing ambiguity over the predominance of the clinical course of cognitive functioning. In particular, contradicting findings in neuroimaging studies suggest that the link between cognition and emotion, is mediated by the clinical course of ADHD. ${ }^{24}$ Although there is a notion that this link also indicates a reverse sequence, the basic premise of neural activity in ADHD is best described by predominant impairments in more lateral prefrontal regions, which fluctuate according to the general clinical course of the disorder itself. ${ }^{61}$

\section{Perspectives in neurocognitive and neuroimaging research in PBD and ADHD}

The current psychiatric research paradigm that seeks to identify neurocognitive models to understand associations between neurobiological dysfunctions, cognitive impairments and clinical symptoms, is gradually changing. Given these neuroimaging and neurocognitive findings, the term "neuroprogression" has been proposed as it encompasses the interplay between neurocognitive and clinical deterioration in PBD. ${ }^{62}$ This conceptualization is supported by findings of reductions in the volume of the corpus callosum and left hippocampus, concurrent increased ventricle volumes, persistent hyper-activation or hypo-activation in ventromedial and dorsolateral prefrontal cortices in patients with earlieronset and multiple mood episodes compared with single-episode populations and later-onset of the disorder. ${ }^{63}$ In similar fashion, findings from longitudinal studies on neurocognitive performance in PBD report that the number of previous manic episodes and successive mood episodes are associated with progressive decline in sustained attention, processing speed and verbal memory. ${ }^{64} \mathrm{It}$ is of great interest that trajectory of functional impairment is evident as early as the prodromal phase of PBD. ${ }^{65}$ Three candidate biological mechanisms have been identified as potential mediators to the neuroprogressive features of BD: neuroplasticity, oxidative stress, and inflammation. ${ }^{66}$ Accounting for neuroplasticity and oxidative stress, brain-derived neurotrophic factor (BDNF) levels are significantly decreased during acute mood episodes, ${ }^{67}$ while elevated DNA oxidation and shortened telomeres are associated with increased frequency of episodes respectively. ${ }^{68}$ Regarding inflammation, BD patients show substantial differences in various chemokines and cytokines, and present with increased levels of interleukin (IL)- 6 during the late stages of the disorder. ${ }^{69,70}$ In contrast with the uni-modal conceptualization of neuroprogression in $\mathrm{BD}$, dual-pathway models, associated with brain abnormalities and subsequent neurocognitive deficits, have been proposed in ADHD. ${ }^{71}$ Based on neuroimaging findings highlighting abnormalities in the reward system (ventral striatum, orbitofrontal cortex) and executive control (lateral medial prefrontal cortices), the dual-pathway models suggest singlecognitive-deficit explanations for different brain systems. ${ }^{72}$ By highlighting neurocognitively defined pathways, fMRI-measured sampling appears to formulate a powerful toolbox for identifying biomarkers within the neurocognitive profile of ADHD.

Despite the advances in the field of neuroimaging in PBD and ADHD, the utility of brain imaging in clinical practice has been hindered by low specificity and sensitivity due to its reliance on group-level statistics. Furthermore, the main criticism targeted at the neuroprogressive model in PBD and dual-pathway model in ADHD is the potential omission of symptomatic subgroups associated with varying degrees of clinical symptoms, demographics and genetic factors. $^{73}$ In light of those limitations, machine learning $(\mathrm{ML})$ combines neuroimaging with pattern identification approaches and tests predictive models in 
large samples providing individual-level results (supervised learning) and defines homogenous groups among heterogeneous samples (unsupervised learning). ${ }^{74}$ By implementing multivariate approaches and intergrading different levels of data (neuroimaging, neurocognitive, genetic, demographics), ML suggests a predictive tool with the potential to deliver outcome biomarkers of PBD and ADHD. ${ }^{75}$ Recently, several studies have demonstrated the ability of $M L$ for differentiating unique BD and ADHD subgroups from control participants, based on neurocognitive and neuroimaging data. ${ }^{76,77}$

\section{Conclusions}

There is a growing body of neurocognitive and neurobiological research that recognizes specific cognitive and emotional patterns in PBD. These observations on the neurobiological mechanisms of cogni- tive impairments in PBD appear to provide robust evidence on a specific neuropsychological profile of PBD, the effect of mood states on neuropsychological functioning and the link between emotion generation and regulation in children diagnosed with PBD. Interestingly, these findings are partially applied in ADHD. Although evidence on specific cognitive impairments suggests a differential neuropsychological profile of ADHD patients, ambiguity still exists about the relationship between clinical course and cognitive impairments, while a distinct phenotype for emotion regulation in these patients is still unknown. Furthermore, ML extends the notion that neuropsychological profiling is biologically relevant and clinically important. These advances dictate the pivotal role of clinical research in raising awareness for PBD but most importantly indicate that there is abundant room for further progress in determining distinct neuropsychological profiles for both PBD and ADHD.

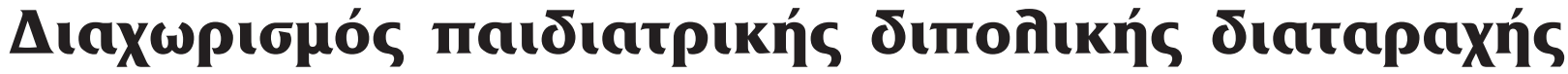

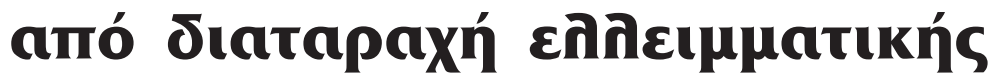

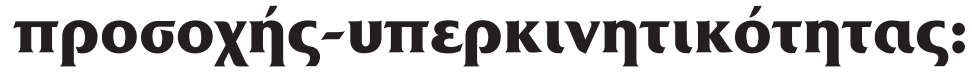

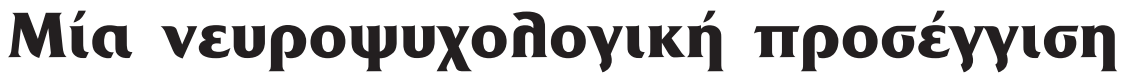

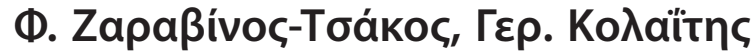

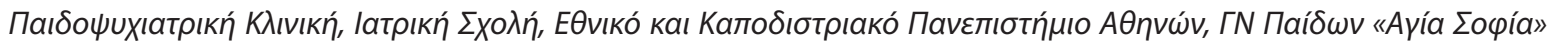

Uuxıатрıкń 2020, 31:332-340

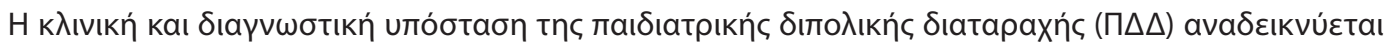

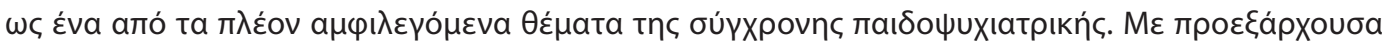

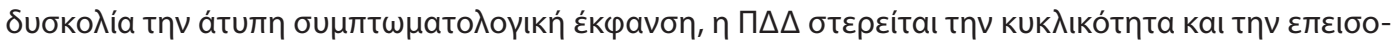

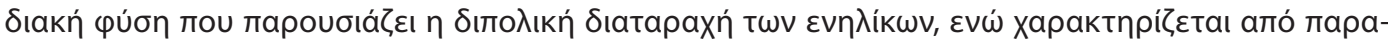

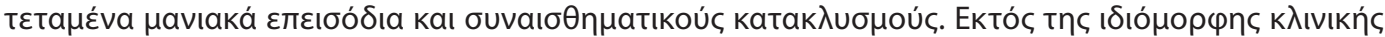

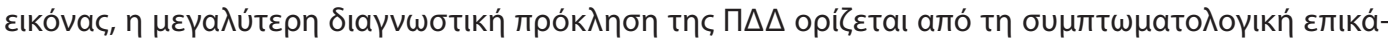

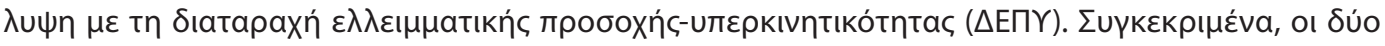

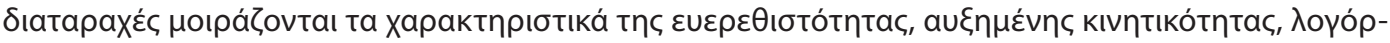

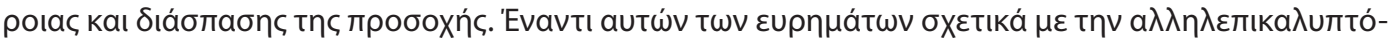

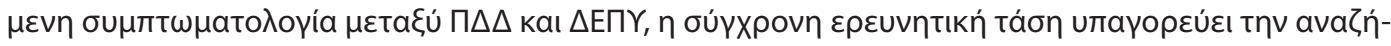

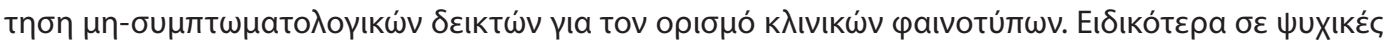

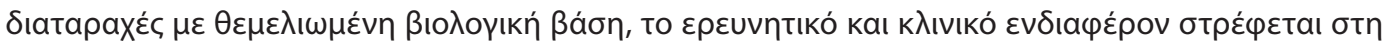

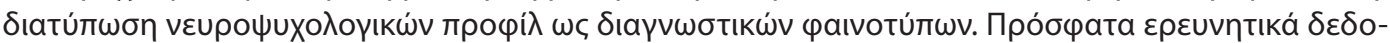

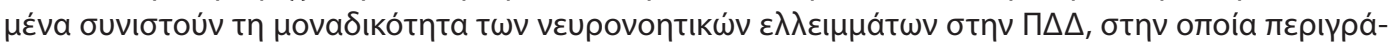




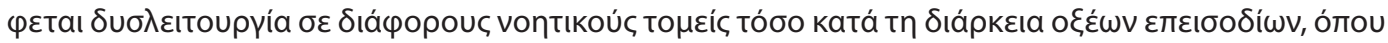

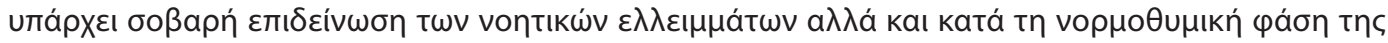

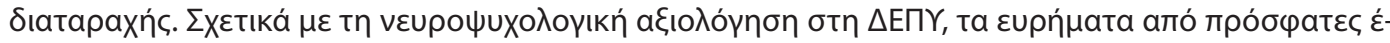

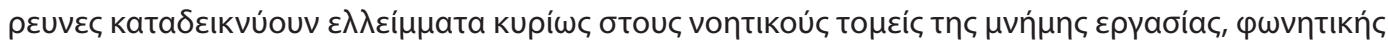

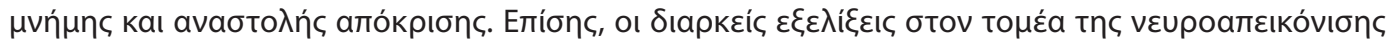

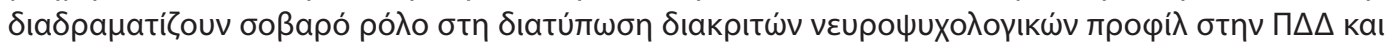

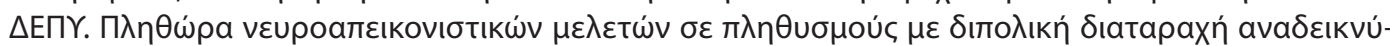

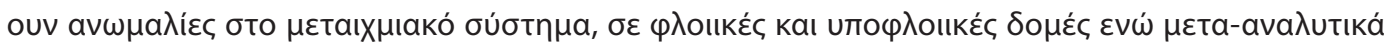

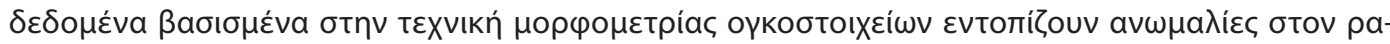

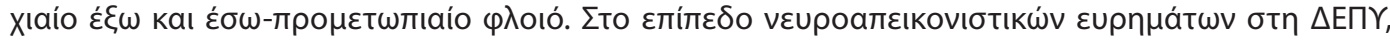

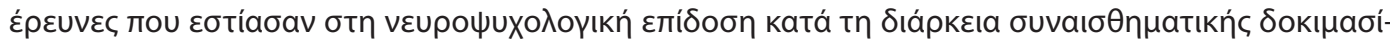

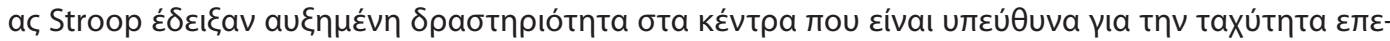

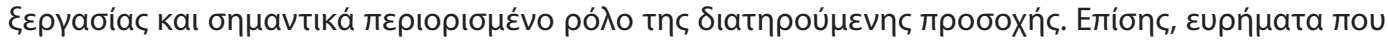

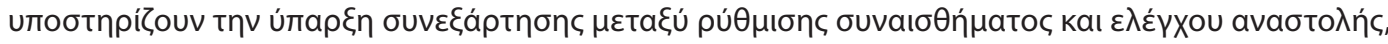

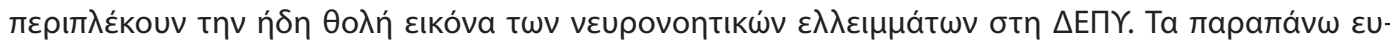

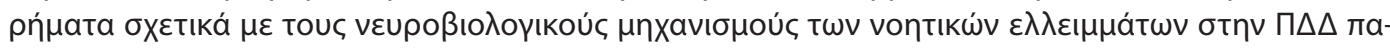

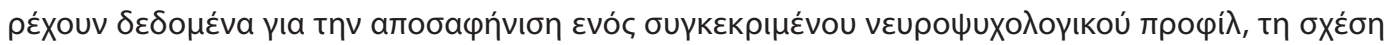

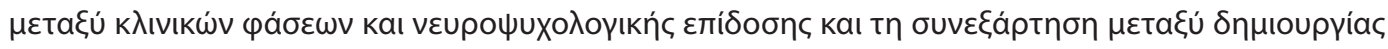

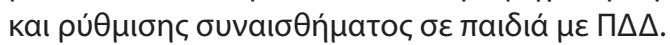

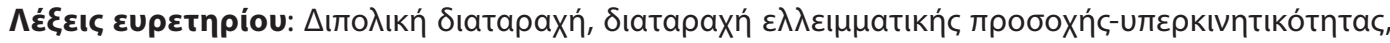

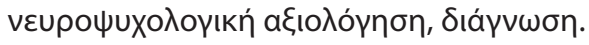

\section{References}

1. Coll PG, Bland R. Manic Depressive Illness in Adolescence and Childhood: Review and Case Report. Can J Psychiatry 1979, 24:255-263, doi: 10.1177/070674377902400311

2. Davis RE. Manic-depressive variant syndrome of childhood: a preliminary report. Am J Psychiatry 1979, 136:702-706, doi: 10.1176/ajp.136.5.702

3. Geller B, Fox LW, Clark KA. Rate and predictors of prepubertal bipolarity during follow-up of 6- to 12-year-old depressed children. J Am Acad Child Adolesc Psychiatry 1994, 33:461-468, doi: 10.1097/00004583-199405000-00003

4. Sylvester CE, Burke PM, McCauley EA, Clark CJ. Manic psychosis in childhood: Report of two cases. J Nerv Ment Dis 1984, 172:12-15, doi: 10.1097/00005053-198401000-00004

5. Tomasson K, Kuperman S. Bipolar disorder in a prepubescent child. J Am Acad Child Adolesc Psychiatry 1990, 29:308-310, doi: 10.1097/00004583-199003000-00023

6. Varanka TM, Weller RA, Weller EB, Fristad MA. Lithium treatment of manic episodes with psychotic features in prepubertal children. Am J Psychiatry 1988, 145:1557-1559, doi: 10.1176/ ajp.145.12.1557

7. Weller RA, Weller EB, Tucker SG, Fristad MA. Mania in prepubertal children: has it been underdiagnosed? J Affect Disord 1986, 11:151-154, doi: 10.1016/0165-0327(86)90022-4

8. Carlson GA. Differential diagnosis of bipolar disorder in children and adolescents. World Psychiatry 2012, 11:146-152, do: 10.1176/ajp.145.12.1557

9. Wozniak J, Biederman J, Kiely K, Ablon JS, Faraone SV, Mundy $\mathrm{E}$ et al. Mania-like symptoms suggestive of childhood-onset bipolar disorder in clinically referred children. J Am Acad Child Adolesc Psychiatry 1995, 34:867-876, doi: 10.1097/00004583199507000-00010
10. Biederman J, Faraone S, Wozniak J, Mick E, Kwon A, Aleardi M. Further evidence of unique developmental phenotypic correlates of pediatric bipolar disorder: findings from a large sample of clinically referred preadolescent children assessed over the last 7 years. $J$ Affect Disord 2004, 82:45-58, doi: 10.1016/j.jad.2004.05.021

11. Carlson GA, Bromet EJ, Sievers S. Phenomenology and outcome of subjects with early- and adult-onset psychotic mania. Am J Psychiatry 2000, 157:213-219, doi: 10.1176/appi.ajp.157.2.213

12. Weckerly J. Pediatric bipolar mood disorder. J Dev Behav Pediatr 2002, 23:42-56, doi: 10.1097/00004703-200202000-00009

13. Pavuluri MN, Birmaher B, Naylor MW. Pediatric bipolar disorder: a review of the past 10 years. J Am Acad Child Adolesc Psychiatry 2005, 44:846-871, doi: 10.1097/01.chi.0000170554.23422.c1

14. Borchardt CM, Bernstein GA. Comorbid disorders in hospitalized bipolar adolescents compared with unipolar depressed adolescents. Child Psychiatry Hum Dev 1995, 26:11-18, doi: 10.1007/ bf02353226

15. West SA, McElroy SL, Strakowski SM, Keck PE, McConville BJ. Attention deficit hyperactivity disorder in adolescent mania. Am J Psychiatry 1995, 152:271-273, doi: 10.1176/ajp.152.2.271

16. Geller B, Zimerman B, Williams M, Delbello MP, Bolhofner K, Craney $\mathrm{JL}$ et al. DSM-IV mania symptoms in a prepubertal and early adolescent bipolar disorder phenotype compared to attention-deficit hyperactive and normal controls. J Child Adolesc Psychopharmacol 2002, 12:11-25, doi: 10.1089/10445460252943533

17. Kim EY, Miklowitz DJ. Childhood mania, attention deficit hyperactivity disorder and conduct disorder: a critical review of diagnostic dilemmas. Bipolar Disord 2002, 4:215-225, doi: 10.1034/j.13995618.2002.01191.x

18. Soutullo CA, Chang KD, Dvez-Suarez A, Figueroa-Quintana A, Escamilla-Canales I, Rapado-Castro $\mathrm{M}$ et al. Bipolar disorder in 
children and adolescents: international perspective on epidemiology and phenomenology. Bipolar Disord 2005, 7:497-506, doi: 10.1111/j.1399-5618.2005.00262.x

19. Van Meter AR, Moreira ALR, Youngstrom EA. Meta-analysis of epidemiologic studies of pediatric bipolar disorder. J Clin Psychiatry 2011, 72:1250-1256, doi: 10.4088/JCP.10m06290

20. Polanczyk G, de Lima MS, Horta BL, Biederman J, Rohde LA. The Worldwide Prevalence of ADHD: A Systematic Review and Metaregression Analysis. AJP 2007, 164:942-948, doi: 10.1176/ ajp.2007.164.6.942

21. Carlson GA. Mania and ADHD: comorbidity or confusion. J Affect Disord 1998, 51:177-187, doi: 10.1016/s0165-0327(98)00179-7

22. Galanter $C A$, Leibenluft E. Frontiers between attention deficit hyperactivity disorder and bipolar disorder. Child Adolesc Psychiatr Clin N Am 2008, 17:325-346, doi: 10.1016/j.chc.2007.11.001

23. Angold A, Costello EJ, Erkanli A. Comorbidity. J Child Psychol Psychiatry 1999, 40:57-87, doi: 10.1111/1469-7610.00424

24. Youngstrom EA, Findling RL, Calabrese JR. Who Are the Comorbid Adolescents? Agreement Between Psychiatric Diagnosis, Youth, Parent, and Teacher Report. J Abnorm Child Psychol 2003, 31:231-245, doi: 10.1023/A:1023244512119

25. Klein RG, Pine DS, Klein DF. Resolved: mania is mistaken for ADHD in prepubertal children. J Am Acad Child Adolesc Psychiatry 1998, 37:1091-1096, doi: 10.1097/00004583-199810000-00020

26. Singh T. Pediatric Bipolar Disorder: Diagnostic Challenges in Identifying Symptoms and Course of Illness. Psychiatry (Edgmont) 2008, 5:34-42, PMID: 19727283

27. Tillman R, Geller B. Definitions of rapid, ultrarapid, and ultradian cycling and of episode duration in pediatric and adult bipolar disorders: a proposal to distinguish episodes from cycles. J Child Adolesc Psychopharmacol 2003, 13:267-271, doi: 10.1089/104454603322572598

28. Hazell PL, Carr V, Lewin TJ, Sly K. Manic symptoms in young males with ADHD predict functioning but not diagnosis after 6 years. J Am Acad Child Adolesc Psychiatry 2003, 42:552-560, doi: 10.1097/01.CHI.0000046830.95464.33

29. Kowatch RA, Fristad M, Birmaher B, Wagner KD, Findling RL, Hellander $\mathrm{M}$ et al. Treatment guidelines for children and adolescents with bipolar disorder. J Am Acad Child Adolesc Psychiatry 2005, 44:213-235, doi: 10.1097/00004583-200503000-00006

30. Mackin P, Targum SD, Kalali A, Rom D, Young AH. Culture and assessment of manic symptoms. Br J Psychiatry 2006, 189:379380, doi: 10.1192/bjp.bp. 105.013920

31. Dubicka B, Carlson GA, Vail A, Harrington R. Prepubertal mania: diagnostic differences between US and UK clinicians. Eur Child Adolesc Psychiatry 2008, 17:153-161, doi: 10.1007/s00787-0070649-5

32. NIMH, National institute of mental health research roundtable on prepubertal bipolardisorder. J Am Acad Child Adolesc Psychiatry 2001, 40:871-878, doi: 10.1097/00004583-200108000-00007

33. Doyle AE, Wilens TE, Kwon A, Seidman LJ, Faraone SV, Fried $R$ et al. Neuropsychological functioning in youth with bipolar disorder. Biol Psychiatry 2005, 58:540-548, doi: 10.1016/j. biopsych.2005.07.019

34. Fleck DE, Shear PK, Zimmerman ME, Getz GE, Corey KB, Jak $A$ et al. Verbal memory in mania: effects of clinical state and task requirements. Bipolar Disord 2003, 5:375-380, doi: 10.1034/j.1399-5618.2003.00055.x
35. Martvnez-Aran A, Vieta E, Reinares M, Colom F, Torrent C, Sanchez-Moreno $\mathrm{J}$ et al. Cognitive function across manic or hypomanic, depressed, and euthymic states in bipolar disorder. Am J Psychiatry 2004, 161:262-270, doi: 10.1176/appi. ajp.161.2.262

36. Robinson LJ, Ferrier IN. Evolution of cognitive impairment in bipolar disorder: a systematic review of cross-sectional evidence. Bipolar Disord 2006, 8:103-116, doi: 10.1111/j.13995618.2006.00277.x

37. Bora E, Yucel M, Pantelis C. Cognitive endophenotypes of bipolar disorder: a meta-analysis of neuropsychological deficits in euthymic patients and their first-degree relatives. $J$ Affect Disord 2009, 113:1-20, doi: 10.1016/j.jad.2008.06.009

38. Walshaw PD, Alloy LB, Sabb FW. Executive Function in Pediatric Bipolar Disorder and Attention-Deficit Hyperactivity Disorder: In Search of Distinct Phenotypic Profiles. Neuropsychol Rev 2010, 20:103-120, doi: 10.1007/s11065-009-9126-x

39. Ferrier IN, Stanton BR, Kelly TP, Scott J. Neuropsychological function in euthymic patients with bipolar disorder. Br J Psychiatry 1999, 175:246-251, doi: 10.1192/bjp.175.3.246

40. Kolur US, Reddy YCJ, John JP, Kandavel T, Jain S. Sustained attention and executive functions in euthymic young people with bipolar disorder. Br J Psychiatry 2006, 189:453-458, doi: 10.1192/ bjp.bp.106.022921

41. Dickstein DP, Milham MP, Nugent AC, Drevets WC, Charney DS, Pine DS et al. Frontotemporal alterations in pediatric bipolar disorder: results of a voxel-based morphometry study. Arch Gen Psychiatry 2005, 62:734-741, doi: 10.1001/archpsyc.62.7.734

42. Barkley RA. Behavioral inhibition, sustained attention, and executive functions: Constructing a unifying theory of ADHD. Psychol Bull 1997, 121:65-94, doi: 10.1037/0033-2909.121.1.65

43. Faraone SV, Biederman J. Neurobiology of attention-deficit hyperactivity disorder. Biol Psychiatry 1998, 44:951-958, doi: 10.1016/ S0006-3223(98)00240-6

44. Torralva T, Gleichgerrcht E, Torrente F, Roca M, Strejilevich SA, Cetkovich $\mathrm{M}$ et al. Neuropsychological functioning in adult bipolar disorder and ADHD patients: a comparative study. Psychiatry Res 2011, 186:261-266, doi: 10.1016/j.psychres.2010.08.007

45. Seidman LJ, Biederman J, Monuteaux MC, Valera E, Doyle AE, Faraone SV. Impact of gender and age on executive functioning: do girls and boys with and without attention deficit hyperactivity disorder differ neuropsychologically in preteen and teenage years? Dev Neuropsychol 2005, 27:79-105, doi: 10.1207/ s15326942dn2701_4

46. Castellanos FX, Lee PP, Sharp W, Jeffries NO, Greenstein DK, Clasen LS et al. Developmental trajectories of brain volume abnormalities in children and adolescents with attention-deficit/ hyperactivity disorder. JAMA 2002, 288:1740-1748, doi: 10.1001/ jama.288.14.1740

47. Carlson CL, Mann M. Attention-Deficit/Hyperactivity Disorder, Predominantly Inattentive Subtype. Child Adolesc Psychiatr Clin N Am 2000, 9:499-510, doi: 10.1016/S1056-4993(18)30103-2

48. Nigg JT, Blaskey LG, Huang-pollock CL, Rappley MD. Neuropsychological Executive Functions and DSM-IV ADHD Subtypes. J Am Acad Child Adolesc Psychiatry 2002, 41:59-66, doi: 10.1097/00004583-200201000-00012

49. Savitz J, Solms M, Ramesar R. Neuropsychological dysfunction in bipolar affective disorder: a critical opinion. Bipolar Disord 2005, 7:216-235, doi: 10.1111/j.1399-5618.2005.00203.x 
50. Altshuler LL, Ventura J, van Gorp WG, Green MF, Theberge DC, Mintz J. Neurocognitive function in clinically stable men with bipolar I disorder or schizophrenia and normal control subjects. Biol Psychiatry 2004, 56:560-569, doi: 10.1016/j. biopsych.2004.08.002

51. Balanza-Martvnez V, Rubio C, Selva-Vera G, Martinez-Aran A, Sanchez-Moreno J, Salazar-Fraile J et al. Neurocognitive endophenotypes (endophenocognitypes) from studies of relatives of bipolar disorder subjects: a systematic review. Neurosci Biobehav Rev 2008, 32:1426-1438, doi: 10.1016/j.neubiorev.2008.05.019

52. Murphy FC, Sahakian BJ. Neuropsychology of bipolar disorder. Br J Psychiatry 2001, 178:120-127, PMID: 11388950

53. Schretlen DJ, Cascella NG, Meyer SM, Kingery LR, Marc Testa $\mathrm{S}$, Munro CA et al. Neuropsychological Functioning in Bipolar Disorder and Schizophrenia. Biol Psychiatry 2007, 62:179-186, doi: 10.1016/j.biopsych.2006.09.025

54. Strakowski SM, Delbello MP, Adler CM. The functional neuroanatomy of bipolar disorder: a review of neuroimaging findings. Mol Psychiatry 2005, 10:105-116, doi: 10.1038/sj.mp.4001585

55. Pavuluri MN, O'Connor MM, Harral E, Sweeney JA. Affective neural circuitry during facial emotion processing in pediatric bipolar disorder. Biol Psychiatry 2007, 62:158-167, doi: 10.1016/j. biopsych.2006.07.011

56. Blumberg HP, Leung H-C, Skudlarski $\mathrm{P}$, Lacadie CM, Fredericks $\mathrm{CA}$, Harris $\mathrm{BC}$ et al. A functional magnetic resonance imaging study of bipolar disorder: state- and trait-related dysfunction in ventral prefrontal cortices. Arch Gen Psychiatry 2003, 60:601-609, doi: 10.1001/archpsyc.60.6.601

57. Chang K, Adleman NE, Dienes K, Simeonova DI, Menon V, Reiss A. Anomalous prefrontal-subcortical activation in familial pediatric bipolar disorder: a functional magnetic resonance imaging investigation. Arch Gen Psychiatry 2004, 61:781-792, doi: 10.1001/ archpsyc.61.8.781

58. Rubinstein JS, Meyer DE, Evans JE. Executive control of cognitive processes in task switching. $J$ Exp Psychol Hum Percept Perform 2001, 27:763-797, doi: 10.1037/0096-1523.27.4.763

59. Shaw P, Stringaris A, Nigg J, Leibenluft E. Emotional dysregulation and Attention-Deficit/Hyperactivity Disorder. Am J Psychiatry 2014, 171:276-293, doi: 10.1176/appi.ajp.2013.13070966

60. Castellanos FX, Proal E. Large-scale brain systems in ADHD: beyond the prefrontal-striatal model. Trends Cogn Sci 2012, 16:17-26, doi: 10.1016/j.tics.2011.11.007

61. Rubia K, Overmeyer S, Taylor E, Brammer M, Williams SC, Simmons $\mathrm{A}$ et al. Hypofrontality in attention deficit hyperactivity disorder during higher-order motor control: a study with functional MRI. Am J Psychiatry 1999, 156:891-896, doi: 10.1176/ ajp.156.6.891

62. Grande I, Berk M, Birmaher B, Vieta E. Bipolar disorder. Lancet 2015, 387:1561-1572, doi: 10.1016/S0140-6736(15)00241-X

63. Stevens MC, Pearlson GD, Calhoun VD, Bessette KL. Functional Neuroimaging Evidence for Distinct Neurobiological Pathways in Attention-Deficit/Hyperactivity Disorder. Biol Psychiatry Cogn Neurosci Neuroimaging 2018, 3:675-685, doi: 10.1016/j. bpsc.2017.09.005

64. Pavuluri MN, West A, Hill SK, Jindal K, \& Sweeney JA. Neurocognitive function in pediatric bipolar disorder: 3-year follow-up shows cognitive development lagging behind healthy youths. J Am Acad Child Adolesc Psychiatry 2009, 48:299-307, doi: 10.1097/CHI.0b013e318196b907
65. Ratheesh A, Lin A, Nelson B, Wood SJ, Brewer W, Betts J et al. Neurocognitive functioning in the prodrome of maniaan exploratory study. J Affect Disord 2013, 147:441-445, doi: 10.1016/j.jad.2012.09.017

66. Berk M. Neuroprogression: pathways to progressive brain changes in bipolar disorder. Int J Neuropsychopharmacol 2009, 12:441-445, doi: 10.1017/S1461145708009498

67. Fernandes BS, Berk M, Turck CW, Steiner J, Gonnalves C-A Decreased peripheral brain-derived neurotrophic factor levels are a biomarker of disease activity in major psychiatric disorders: a comparative meta-analysis. Mol Psychiatry 2014, 19:750-751, doi: $10.1038 / \mathrm{mp} .2013 .172$

68. Soeiro-de-Souza MG, Andreazza AC, Carvalho AF, MachadoVieira R, Young LT, Moreno RA. Number of manic episodes is associated with elevated DNA oxidation in bipolar I disorder. Int J Neuropsychopharmacol 2013, 16:1505-1512, doi: 10.1017/ S1461145713000047

69. Panizzutti B, Gubert C, Schuh AL, Ferrari P, Bristot G, Fries GR et al. Increased serum levels of eotaxin/CCL11 in late-stage patients with bipolar disorder: An accelerated aging biomarker? J Affect Disord 2015 182:64-69, doi: 10.1016/j.jad.2014.12.010

70. Kauer-Sant'Anna M, Kapczinski F, Andreazza AC, Bond DJ, Lam RW, Young LT et al. Brain-derived neurotrophic factor and inflammatory markers in patients with early-vs. late-stage bipolar disorder. Int J Neuropsychopharmacol 2009, 12:447-458, doi: $10.1017 / \mathrm{S} 1461145708009310$

71. Sonuga-Barke EJS. The dual pathway model of AD/HD: an elaboration of neuro-developmental characteristics. Neurosci Biobehav Rev 2003, 27:593-604, doi: 10.1016/j.neubiorev.2003.08.005

72. McCarthy H, Skokauskas N, Frodl T. Identifying a consistent pattern of neural function in attention deficit hyperactivity disorder: a meta-analysis. Psychol Med 2014, 44:869-880, doi: 10.1017/ S0033291713001037

73. Phillips ML, Swartz HA. A Critical Appraisal of Neuroimaging Studies of Bipolar Disorder: Toward a New Conceptualization of Underlying Neural Circuitry and a Road Map for Future Research. AJP 2014, 171:829-843, doi: 10.1176/appi.ajp.2014.13081008

74. Claude L-A, Houenou J, Duchesnay E, Favre P. Will machine learning applied to neuroimaging in bipolar disorder help the clinician? A critical review and methodological suggestions. Bipolar Disord 2020, 22:334-355, doi: 10.1111/bdi.12895

75. Passos IC, Mwangi B, Kapczinski F. Big data analytics and machine learning: 2015 and beyond. Lancet Psychiatry 2016, 3:13-15, doi: 10.1016/S2215-0366(15)00549-0

76. Achalia R, Sinha A, Jacob A, Achalia G, Kaginalkar V, Venkatasubramanian $\mathrm{G}$ et al. A proof of concept machine learning analysis using multimodal neuroimaging and neurocognitive measures as predictive biomarker in bipolar disorder. Asian $J$ Psychiatr 2020, 50:101984, doi: 10.1016/j.ajp.2020.101984

77. Hart H, Chantiluke K, Cubillo AI, Smith AB, Simmons A, Brammer $M J$ et al. Pattern classification of response inhibition in ADHD: Toward the development of neurobiological markers for ADHD. Hum Brain Mapp 2013, 35:3083-3094, doi: 10.1002/hbm.22386

Corresponding author: F. Zaravinos-Tsakos, Department of Child Psychiatry, School of Medicine, National and Kapodistrian University of Athens, "Aghia Sophia" Children's Hospital, Thivon \& Papadiamantopoulou, GR-115 27 Athens, Greece e-mail:ph.zarts@gmail.com 